\title{
Records and 2-block records of 1-dependent stationary sequences under local dependence
}

\author{
by \\ George HAIMAN \\ Université de Lille 1 and URA CNRS 1321. \\ Nelly MAYEUR \\ Université de Lille 1. \\ Valéry NEVZOROV \\ St. Petersburg University. \\ and \\ Madan L. PURI \\ Indiana University.
}

ABstRact. - We first study the records of several examples of 1dependent stationary sequences $\left\{X_{n}\right\}$, some of them which satisfy a local independence condition introduced in Haiman (1987a), whereas the other do not satisfy this condition.

For the second category (called with local dependence), we then extend a result of the above cited paper by proving, (under some regularity conditions on the joint distribution of $\left.\left(X_{1}, X_{2}, X_{3}\right)\right)$, that the 2-block record times of $\left\{X_{n}\right\}$ a.s. coincide, (via a translation of the time index and from some index on) with the record times of an appropriately constructed i.i.d. sequence $\left\{X_{n}\right\}$. whereas the 2-block record values of $\left\{X_{n}\right\}$ and the record values of $\left\{\hat{X}_{n}\right\}$ are imbricate. (c) Elsevier, Paris

Key words and phrases: 1-dependent stationary sequences. records. 2-block records. clusters, local dependence, strong approximation, extremes.

AMS Classifications: $60 \mathrm{~F} 15,60 \mathrm{G} 15,62 \mathrm{G} 30$.

Alnates de l'hstitut Henri Poincaré - Probabilités et Statistiques - 1246-(12013.3

Vol. 34/98/14/G Elsevier. Paris 
RÉSUMÉ. - On étudie d'abord les records pour plusieurs exemples de suites stationnaires 1-dépendantes $\left\{X_{n}\right\}$, certaines satisfaisant une condition d'indépendance locale introduite dans Haiman (1987a) et d'autres ne la satisfaisant pas. Pour la deuxième catégorie nous étendons un résultat de l'article précité en démontrant (sous des conditions de régularité portant sur la loi de $\left.\left(X_{1}, X_{2}, X_{3}\right)\right)$ que les instantes de records par blocs de deux de $\left\{X_{n}\right\}$ cö̈ncident presque sûrement (modulo une translation d'indice et à partir d'un certain rang) avec les instants de records d'une suite i.i.d. $\left\{\hat{X}_{n}\right\}$ convenablement construite, alors que les valeurs de records par blocs de deux de $\left\{X_{n}\right\}$ et les records de $\left\{\hat{X}_{n}\right\}$ sont imbriqués. (C) Elsevier, Paris

\section{INTRODUCTION}

Let $\left\{X_{n}\right\}_{n \geq 1}$, be a strictly stationary sequence of random variables (r.v.'s) with continuous marginal d.f. $F(x)=P\left\{X_{1} \leq x\right\}$. Many papers have been devoted to the study of the records of $\left\{X_{n}\right\}_{n \geq 1}$, especially when $\left\{X_{n}\right\}_{n \geq 1}$ is i.i.d.. For an overview of these results, the reader is referred to Nevzorov (1987) and the references cited therein.

The classical formal definition of record times $T_{n}$ and record values $\theta_{n}$ is:

$$
T_{1}=1 . \theta_{1}=X_{1}
$$

and for $n \geq 1$,

$$
T_{n+1}=\inf \left\{k>T_{n}, X_{k}>\theta_{n}\right\} \text { and } \theta_{n+1}=X_{T_{n+1}} .
$$

However, in the sequel we will use the following more general definition of records with respect to an initial threshold $\theta_{0}, \alpha<\theta_{0}<\omega$, where $\alpha=\inf \{x ; F(x)>0\}$ and $\omega=\sup \{x ; F(x)<1\}$, (the left and right end points of $F$ ).

DEFinition 1.1. -- (Haiman (1987a))

$$
T_{1}=\inf \left\{k \geq 1: X_{k}>\theta_{0}\right\}, \theta_{1}=X_{T_{1}}
$$

and for $n \geq 1$

$$
T_{n+1}=\inf \left\{k>T_{n} . X_{k}>\theta_{n}\right\} \text { and } \theta_{n+1}=X_{T_{n+1}} .
$$


This definition is justified by the following proposition:

PROPOSITION 1.1. - (Haiman (1987a))

If $\left\{\left(T_{n}^{\prime}, \theta_{n}^{\prime}\right)\right\}$ is any other sequence such that for $n \geq n_{0}$

$$
T_{n+1}^{\prime}=\inf \left\{k>T_{n}^{\prime} . X_{k}>\theta_{n}^{\prime}\right\} \text { and } \theta_{n+1}^{\prime}=X_{T_{n+1}^{\prime}},
$$

then there exist $n_{1}$ and $q$ such that for $n \geq n_{1}$, we have

$$
T_{n}^{\prime}=T_{n-q} \text { and } \theta_{n}^{\prime}=\theta_{n-t} .
$$

For m-dependent sequences (i.e. for any $t \geq 1, \sigma\left(\ldots X_{t}\right)$ and $\sigma\left(X_{t+m-1}, \ldots\right)$ are independent $)$, the following theorem was obtained in (Haiman (1987a)). under the additional "local independence" hypothesis:

There exists a $\beta>0$ such that for $2 \leq k \leq m+1$

$$
\limsup _{=/ \omega}\left\{\sup _{\substack{z<u \\ z<t}} P\left\{X_{1}>u \mid X_{k}=v\right\}\left(P\left\{X_{1}>u\right\}\right)^{-i}\right\}<\alpha .
$$

THEOREM 1.1. - Let $\left\{X_{n}\right\}_{n \geq 1}$ satisfy hypothesis (1.2). Then there exists a probability space which carries, in addition to $\left\{X_{n}\right\}_{n \geq 1}$, an i.i.d. sequence $\left\{X_{n}\right\}_{n \geq 1}$ having the same marginal distribution and such that. if $\left\{\left(\hat{T}_{n}, \hat{\theta}_{n}\right)\right\}_{n \geq 1}$ are the records of $\left\{\hat{X}_{n}\right\}_{n \geq 1}$, then a.s. there exist two r.v.' $s$ $N_{0}$ and $Q$ such that for $n \geq N_{0}$.

$$
T_{n}=\hat{T}_{n-Q} \text { and } \theta_{n}=\hat{\theta}_{n-Q} \text {. }
$$

The method of proof of theorem 1.1 was adapted in order to obtain the same statement (1.3) for some classes of Gaussian stationary sequences (Haiman (1987 b) and Haiman and Puri (1993)) and also for some Markov sequences (Haiman, Kiki and Puri (1994)). A multivariate version of theorem 1.1 was obtained in Haiman (1992).

Let now $\left\{X_{n}\right\}_{n \geq 1}$ be any sequence of r.v.'s for which the record times $T_{n}$ defined in (1.1) are a.s. finite for any $n \geq 1$. Let $\theta_{n}$ be the corresponding record values. Consider another sequence $\left\{\hat{X}_{n}\right\}_{n \geq 1}$ whose records $\left(\hat{T}_{n}, \hat{\theta}_{n}\right)$ are similarly defined a.s. for any $n \geq 1$. Let

$$
M_{n}=\max \left(X_{1}, \ldots, X_{n}\right)
$$

and

$$
\hat{M}_{n}=\max \left(\hat{X}_{1} \ldots, \hat{X}_{n}\right), n \geq 1 .
$$

It is clear that the sequences $\left\{\left(T_{n}, \theta_{n}\right)\right\}_{n \geq 1}$ and $\left\{\left(\dot{T}_{n}, \dot{\theta}_{n}\right)\right\}_{n \geq 1}$ completely determine the sequences $\left\{M_{n}\right\}_{n>1}$ and $\left\{\hat{M}_{n}\right\}_{n>1}$. Then, elementary Vol. 34. $n^{\circ}+1998$ 
arguments based on the monotonicity of the sequences $\left\{\theta_{n}\right\}_{n \geq 1}$ and $\left\{\hat{\theta}_{n}\right\}_{n \geq 1}$ lead to the following theorem 1.2 (we improve here the statements of Haiman (1992), th. 1.1, p.147 and Haiman and Puri (1993), th.1.1, p.88):

THEOREM 1.2. - If there exist r.v.'s $N_{0}$ and $Q$ such that a.s. for $n \geq N_{0}$. (1.3) is satisfied, then there exists a random variable $N_{1}$ such that for $n \geq N_{1}$, we have

$$
M_{n}=\hat{M}_{n} \text { a.s. }
$$

It may be seen (Haiman (1987a), p. 437) that the proof of theorem 1.1 in the general case $(m \geq 1)$ easily follows from the proof in the case $m=1$, where (1.2) becomes

$$
\limsup _{z / \omega}\left\{\sup _{\substack{c<u \\ \Sigma<u}} P\left\{X_{1}>u \mid X_{2}=v\right\}\left(P\left\{X_{1}>u\right\}\right)^{-3}\right\}<\infty .
$$

It also may be seen that for $m=1$ condition (1.5) may be replaced by the weaker condition: there exists $\beta>0$ and $k>0$ such that

$$
\limsup _{u / \omega}\left\{\sup _{u<u<\psi(u)} P\left\{X_{1}>u \mid X_{2}=v\right\}\left(P\left\{X_{1}>u\right\}\right)^{-i t}\right\}<\infty
$$

where $\varphi(u)$ is the solution of the equation

$$
1-F(\varphi(u))=(1-F(u))^{1+k} .
$$

This is a consequence of the construction method used in Haiman (1987a) and the fact (see Haiman and Puri (1993), lemma 3.3. p. 122) that when $\left\{X_{n}\right\}_{n \geq 1}$ is i.i.d., we have, for any $k>0$,

$$
P\left\{1-F\left(\theta_{n+1}\right)<\left(1-F\left(\theta_{n}\right)\right)^{k} \text { i.o. }\right\}=0 .
$$

The paper is constructed as follows: in section 2, we study the records of several examples of 1 -dependent sequences $\left\{X_{n}\right\}_{n \geq 1}$, some of them which satisfy condition (1.6) whereas the other do not satisfy this condition. We call sequences belonging to the second category "with local dependence" and show (example 7) that the records of such sequences may behave very different from the records of i.i.d. sequences.

In section 3, we consider sequences which do not necessarly satisfy condition (1.6). We show that under some regularity conditions (satisfied by all our examples) a general version of theorem 1.1 (theorem 3.1), may be obtained. 


\section{STUDY OF EXAMPLES}

Let $(\mathrm{E}, \mathcal{E})$ be some measurable space and $f: \mathrm{E}^{2} \rightarrow \mathbb{R}$ some measurable function. The most classical examples of 1-dependent stationary sequences are of the form

$$
X_{n}=f\left(\xi_{n}, \xi_{n+1}\right), n \geq 1
$$

where $\left\{\xi_{n}\right\}_{n \geq 1}$ is a sequence of i.i.d. (E, $\left.\mathcal{E}\right)$-valued random elements.

Our examples are all of this form. However, "exotic" examples which do not have a representation like (2.1), (for a recent discussion on this subject, see for example, Matus (1993)) may be given.

The following examples 1-4 satisfy condition (1.6).

\subsection{Example 1}

Let $\left\{Y_{n}\right\}_{n \geq 1}$ be a sequence of independent $\mathcal{N}(0,1)$ normally distributed r.v.'s and let

$$
X_{n}=a Y_{n}+b Y_{n+1}, \quad n \geq 1,
$$

where $a$ and $b$ are two constants such that $0<|a|<1$ and $a^{2}+b^{2}=1$. It may be shown (see Mayeur (1996)) that if $\rho=\operatorname{Cov}\left(X_{n}, X_{n+1}\right)=a b<0$, then $\left\{X_{n}\right\}_{n \geq 1}$ satisfies condition (1.5) whereas if $\rho>0$, it does not satisfy this condition but satisfies condition (1.6) for any $h:>0$.

\subsection{Examples 2, 3 and 4}

Let $\left\{U_{n}\right\}_{n \geq 1}$ be a sequence of i.i.d. uniformly on $[0,1]$ distributed r.v.'s. We now consider examples of the form $X_{n}=\varphi\left(U_{n}, U_{n+1}\right)$ and show that these examples satisfy the following condition, which in turn implies (1.6): There exists $\beta>0$ such that

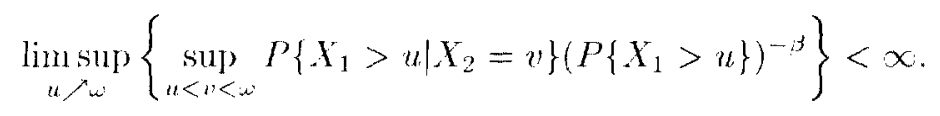

2.2.1. Example 2. $-X_{n}=U_{n}+U_{n+1}, \quad n \geq 1$.

By elementary calculations, we obtain

$$
P\left\{X_{1} \leq x\right\}- \begin{cases}0 & \text { if } x \leq 0 \\ x^{2} / 2 & \text { if } 0 \leq x \leq 1 \\ -x^{2} / 2+2 x-1 & \text { if } 1 \leq x \leq 2 \\ 1 & \text { if } x \geq 2\end{cases}
$$

Vol. 34. $n^{2}+1998$. 
and, for $1 \leq u \leq n \leq 2$.

$$
\begin{aligned}
P\left\{X_{1} \leq u . X_{2} \leq v\right\}= & (u-1)+u(u-u)-\frac{1}{2}(v-1)^{2}+\frac{1}{2}(u-1)^{2} \\
& +u v(2-v)-\frac{1}{2}(u+v)\left[1-(v-1)^{2}\right] \\
& +\frac{1}{3}\left[1-(u-1)^{3}\right] .
\end{aligned}
$$

Note that since all our examples are of the form $X_{n}=\varphi\left(U_{n}, U_{n+1}\right)$, we have

$$
\begin{aligned}
P\left\{X_{1} \leq u, X_{2} \leq u\right\} & =\int_{0}^{1} P\left\{\varphi\left(U_{1}, U_{2}\right) \leq u \cdot \varphi\left(U_{2}, U_{3}\right) \leq \eta \mid U_{2}=x\right\} d x \\
& =\int_{11}^{1} P\left\{\varphi\left(U_{1}, r\right) \leq u\right\} \times P\left\{\varphi\left(x, U_{3}\right) \leq v\right\} d x
\end{aligned}
$$

Combining (2.4) and (2.5), for $1 \leq u \leq v \leq 2$, we obtain

$$
\begin{aligned}
P\left\{X_{1}>u \mid X_{2}=u\right\} & =\frac{d}{d u} P\left\{X_{1}>u . X_{2}<v\right\} / \frac{d}{d v} P\left\{X_{2}<n\right\} \\
& =\frac{1}{2}(2+v)-u .
\end{aligned}
$$

Next, if we put $u=2-\varepsilon, v=2-\eta, 0 \leq \eta \leq \varepsilon \leq 1$, (here $\omega=2$ ). we get

$$
\frac{P\left\{X_{1}>u \mid X_{2}=v\right\}}{\left(P\left\{X_{1}>u\right\}\right)^{s}}=2 \frac{\varepsilon-\frac{1}{2} \eta}{\varepsilon^{2 ;}}
$$

which is bounded by $2^{1 / 2}$ for $\beta=\frac{1}{2}$. Thus, $(2.3)$ is satisfied.

2.2.2. Example 3. $-X_{n}=U_{n} \times U_{n+1} . \quad n \geq 1$.

We obtain

$$
P\left\{X_{1} \leq x\right\}= \begin{cases}0 & \text { if } x \leq 0 \\ x(1-\log x) & \text { if } 0 \leq x \leq 1 \\ 1 & \text { if } x \geq 1\end{cases}
$$

and for $0 \leq u \leq v \leq 1$,

$$
\begin{aligned}
& P\left\{X_{1} \leq u X_{2} \leq u\right\}=u+u(\log w-\log u)+u-u v . \\
& \text { Anules de Thstitur Henri Poncaré - Probabilités et Statistiques }
\end{aligned}
$$


Thus, if we put $u=1 \cdots \varepsilon \cdot n=1-\eta, 0<\eta<\varepsilon<1$, (here $\omega=1$ ), we get

$$
\begin{aligned}
\frac{P\left\{X_{1}>u \mid X_{2}-v\right\}}{\left(P\left\{X_{1}>u\right\}\right)^{3}} & =\frac{-\log v+u-u}{(1-u+u \log u)^{3}(-\log u)} \\
& \sim 2 \cdot \frac{\varepsilon-\frac{1}{2} \eta}{\varepsilon^{2}} \cdot \varepsilon \cdot \eta \rightarrow 0 .
\end{aligned}
$$

which coincides with the last term in (2.7).

2.2.3. Example 4. $-X_{n}=\inf \left(U_{n} \cdot U_{n+1}\right) . \quad n \geq 1$.

We obtain

$$
P\left\{\mathrm{X}_{1} \leq x\right\}= \begin{cases}0 & \text { if } x \leq 0 \\ 2 x-x^{2} & \text { if } 0 \leq x \leq 1 \\ 1 & \text { if } x \geq 1\end{cases}
$$

and for $0 \leq u \leq v \leq 1$.

$$
P\left\{X_{1} \leq u, X_{2} \leq u\right\}=u(1-u)+u v(2-u) .
$$

Thus, if we put $u=1-\varepsilon, n=1-\eta, 0<\eta<\varepsilon<1$, we get

$$
\frac{P\left\{X_{1}>u \mid X_{2}=n\right\}}{\left(P\left\{X_{1}>u\right\}\right)^{3}}=\frac{1-v-u+u n}{\left(1-2 u+u^{2}\right)^{3}(1-\eta)}=\frac{1}{s^{21-1}}
$$

which equals 1 if $\beta=\frac{1}{2}$. Thus (2.3) is satisfied.

The following examples 5,6 and 7 do not satisfy condition (1.6).

\subsection{Example 5}

Let again $\left\{U_{n}\right\}_{n \geq 1}$ be a sequence of i.i.d. uniformly on $[0,1]$ distributed r.v.'s. Let

$$
X_{n}=\max \left(U_{n}, U_{n+1}\right), n \geq 1 .
$$

We obtain

$$
P\left\{X_{1} \leq x\right\}= \begin{cases}0 & \text { if } x \leq 0 \\ x^{2} & \text { if } 0 \leq x \leq 1 \\ 1 & \text { if } x \geq 1\end{cases}
$$

and

$$
P\left\{X_{1} \leq u, X_{2} \leq v\right\}=u^{2} \eta, 0 \leq u \leq v \leq 1 .
$$

Vol. 34, $n^{\circ} 4-1998$ 
Thus

$$
\frac{P\left\{X_{1}>u, X_{2}>u\right\}}{P\left\{X_{1}>u\right\}}-\frac{1-2 u^{2}+u^{3}}{1-u^{2}}=1-\frac{u^{2}}{1+u}-\frac{1}{2}, u \rightarrow 1 .
$$

Condition (1.6) is not satisfied since it implies

$$
\lim _{u \rightarrow \infty} \frac{P\left\{X_{1}>u, X_{2}>u\right\}}{P\left\{X_{1}>u\right\}}=0 .
$$

Remark 2.1. - In this example, the records of $\left\{X_{n}\right\}_{n \geq 1}$ coincide with the records of $\left\{U_{n+1}\right\}_{n}$. Thus, the sequence $\left\{U_{n+1}\right\}_{n}$ trivially satisfies the requirements of $\left\{\hat{X}_{n}\right\}_{n \geq 1}$ in theorem 1.1. This shows that condition (1.6) in this theorem is sufficient but not necessary. (See also remark 3.5).

\subsection{Example 6}

Let $\left\{Z_{n}\right\}_{n \geq 0}$ be a sequence of i.i.d. exponentially with parameter 1 distributed r.v.'s and $\left\{J_{n}\right\}_{n \geq 1}$ a sequence of i.i.d. Bernoulli $\mathcal{B}(1, p)$ r.v.'s. We suppose $\left\{Z_{n}\right\}_{n \geq 0}$ and $\left\{J_{n}\right\}_{n \geq 1}$ independent. Let

$$
X_{n}= \begin{cases}Z_{n} & \text { if } J_{n}=0 \\ Z_{n-1} & \text { if } J_{n}=1 .\end{cases}
$$

The sequence $\left\{X_{n}\right\}_{n \geq 1}$ is of the form (2.1), hence it is stationary 1dependent.

We obtain

$$
F(x)=P\left\{X_{1} \leq x\right\}=1-e^{-x}
$$

and, for $x_{1}, x_{2} \geq 0$,

$$
P\left\{X_{1} \leq x_{1}, X_{2} \leq x_{2}\right\}=(1-q) F\left(x_{1}\right) F\left(x_{2}\right)+q \psi\left(x_{1}, x_{2}\right) .
$$

where $q=p(1-p)$ and $\psi\left(x_{1}, x_{2}\right)=1-p^{-\inf \left(x_{1}, x_{2}\right)}$

Thus,

$$
\lim _{u \rightarrow \infty} \frac{P\left\{X_{1}>u, X_{2}>u\right\}}{P\left\{X_{1}>u\right\}}=\lim _{u \rightarrow \infty} \frac{q c^{-u}+(1-q) e^{-2 u}}{e^{-u}}=q>0 .
$$

\subsection{Example 7}

Let $\left\{Z_{n}\right\}_{n \geq n}$ and $\left\{J_{n}\right\}_{n \geq 1}$ be as in example 6 and let $\left\{V_{n}\right\}_{n \geq 1}$ be a sequence of i.i.d. uniformly on $[0,1]$ distributed r.v.'s, independent of $\left\{Z_{n}\right\}_{n \geq 0}$ and $\left\{J_{n}\right\}_{n \geq 1}$. Let

$$
X_{n}= \begin{cases}Z_{n} & \text { if } J_{n}=0 \\ Z_{n-1}+V_{n} & \text { if } J_{n}=1\end{cases}
$$


We then obtain (for further details, see Mayeur (1996), p. 47), for $x \geq 1$,

$$
P\left\{X_{1}>x\right\}=e^{-x}(1+p(e-2)) \text {. }
$$

and

$P\left\{X_{1}>x, X_{2}>r\right\}=e^{-x} p(1-p)+e^{-2 x}\left[(1+p(e-2))^{2}-p(1-p)(e-1)\right]$.

Thus

$$
\lim _{r \rightarrow \infty} \frac{P\left\{X_{1}>x, X_{2}>x\right\}}{P\left\{X_{1}>x\right\}}=\frac{p(1-p)}{1+p(e-2)}>0 .
$$

Remark 2.2. - The record values $\left\{\theta_{n}\right\}$ of $\left\{X_{n}\right\}$ in example 6 behave as the record values of an i.i.d. sequence of exponential $E(1)$ r.v.'s (i.e. $\theta_{n+1}-\theta_{n}, n \geq 1$ form a sequence of i.i.d. exponential $E(1)$ r.v.'s, (see Nevzorov (1987))). As $n \rightarrow \infty$, the number $N(n)$ of records in $X_{1}, \ldots, X_{n}$ is approximately the same as in a sample of $n p(1-p)$ i.i.d. r.v.'s (i.e. $\mathbb{E}(N) \sim \log n p(1-p), n \rightarrow \infty)$.

Remark 2.3. - The record times $\left\{T_{n}\right\}$ of $\left\{X_{n}\right\}$ in example 7 have the particularity that

$$
P\left\{T_{n+1}-T_{n}=1 \text { i.o. }\right\}=1
$$

whereas the record times of an i.i.d. sequence are such that

$$
P\left\{T_{n+1}-T_{n} \nearrow \infty\right\}=1 \text {. }
$$

However, an easy consequence of theorem 3.1 (section 3) is that, for any $k \geq 2$, we have

$$
P\left\{T_{n+1}-T_{n}=k: i . o .\right\}=0 .
$$

The proof of (2.12) follows from the fact (see also remark 2.2) that

$$
\begin{gathered}
P\left\{\left(J_{T_{n}^{\prime}}=0 . J_{T_{n+1}^{\prime \prime}}=1 . J_{T_{n+1}^{z}}=0 . J_{T_{n+1}^{Z}+1}=1\right)\right. \\
\left.\cap\left(\theta_{n+1}^{Z}-\theta_{n}^{Z}>1\right) \text { i.o. }\right\}=1,
\end{gathered}
$$

where $\left\{\left(T_{n}^{Z}, \theta_{n}^{Z}\right)\right\}$ is the sequence of records of $\left\{Z_{n}\right\}_{n \geq 1}$ in (2.11). Note that when an event inside the brackets of (2.15) occurs, there is a $k \geq 1$ such that $T_{k}=T_{n+1}^{Z}$ and $T_{k+1}-T_{k}=1$. For further details, see Mayeur (1996), p. 49. 
Remark 2.4. - Observe that in examples 5,6 and 7, we have

$$
\lim _{x \rightarrow \infty} \frac{P\left\{X_{1}>u, X_{2}>u\right\}}{P\left\{X_{1}>u\right\}}=\alpha
$$

with $0<\alpha \leq \frac{1}{2}$. We close this section by prooving the following proposition 2.1 .

PROPOSITION 2.1. - If $\left\{X_{n}\right\}$ is a 1 -dependent stationary sequence such that (2.16) is satisfied for some $\alpha>0$, then $\alpha \leq \frac{1}{2}$.

Proof. - The limit in (2.16) is $\alpha, 0 \leq r \leq 1$, if and only if

$$
\lim _{u \rightarrow \infty} \frac{P\left\{X_{1}>u \cdot X_{2} \leq u\right\}}{P\left\{X_{1}>u\right\}}=1-\imath \text {. }
$$

Next, using 1-dependence and stationarity.

$$
\begin{aligned}
& \frac{P\left\{X_{1}>u . X_{2}>u, X_{3}>u\right\}}{P\left\{X_{1}>u\right\}} \\
& \quad=\frac{P\left\{X_{1}>u \cdot X_{2}>u\right\}}{P\left\{X_{1}>u\right\}}-\frac{P\left\{X_{1}>u, X_{2}>u . X_{3} \leq u\right\}}{P\left\{X_{1}>u\right\}} \\
& \quad \leq \frac{P\left\{X_{1}>u X_{3}>u\right\}}{P\left\{X_{1}>u\right\}}=I\left\{X_{1}>u\right\} \underset{u \rightarrow \omega}{\longrightarrow} 0 .
\end{aligned}
$$

where

$$
\frac{P\left\{X_{1}>u, X_{2}>u, X_{3} \leq u\right\}}{P\left\{X_{1}>u\right\}} \leq \frac{P\left\{X_{1}>u . X_{2} \leq u\right\}}{P\left\{X_{1}>u\right\}} \underset{u \rightarrow \infty}{\longrightarrow} 1-\imath .
$$

But, by (2.17), the limit as $u \rightarrow \omega$ of the left hand term in (2.18) must be $x$. Thus $\alpha \leq \frac{1}{2}$.

\section{EXTENSION OF THEOREM 1.1.}

We now consider a 1-dependent sequence $\left\{X_{n}\right\}_{n \geq 1}$ with continuous marginal d.f. $F(x)=P\left\{X_{1} \leq x\right\}$, which we suppose strictly increasing near the right end point $\omega$ of $X_{1}$, i.e. for $x \geq x_{0}, x_{0}<\omega$. Furthermore, we suppose that $\left\{X_{n}\right\}_{n \geq 1}$ satisfies the following hypotheses $\mathbf{H} 1$ and $\mathbf{H}$ :

H1.) The function $\check{H}(u)=P\left\{F\left(X_{1}\right)>u . F\left(X_{2}\right)>u\right\}$ is continuously differentiable near $u=1$ and

$$
\dot{H}^{\prime}(1)=\lim _{x \rightarrow \infty} \frac{P\left\{X_{1}>x, X_{2}>x\right\}}{P\left\{X_{1}>x\right\}}=\alpha, 0 \leq \alpha \leq 1 / 2 .
$$


H2.) There exists a constant $K>0$ such that for $u \leq v \leq \omega$, we have

$$
\begin{aligned}
& P\left\{X_{2}>u, \max \left(X_{2}, X_{3}\right) \in(v, v+d v)\right\} \\
& \quad \leq K P\left\{X_{1} \leq u, X_{2}>u, \max \left(X_{2}, X_{3}\right) \in(v, v+d v)\right\} .
\end{aligned}
$$

Remark 3.1. - It is rather easy to check, (even if for example 7 some calculations are quite lenghly) that all examples satisfy hypotheses $\mathbf{H} \mathbf{1}$ and H2. If we compare condition (I.6) of theorem 1.1 and $\mathbf{H 1}-\mathrm{H} 2, \mathrm{H} 1$ is weaker than (1.6) and then $\mathbf{H} \mathbf{2}$ appears as a compensation of this fact.

Remark 3.2. - Consider the function

$$
\begin{aligned}
H(u): & =P\left\{F\left(X_{1}\right) \leq u, F\left(X_{2}\right)>u\right\} \\
& =P\left\{F\left(X_{1}\right)>u\right\}-\tilde{H}(u) \\
& =1-u-\dot{H}(u) . \quad F\left(x_{0}\right)<u \leq 1 .
\end{aligned}
$$

Hypothesis $\mathbf{H 1}$ implies that there exists $u_{0}, F\left(x_{0}\right) \leq u_{0}<1$. such that $H(u)$ is strictly decreasing to zero for $u_{t} \leq u \leq 1$. However, examples of 1-dependent stationary sequences for which such an $u_{0}$ does not exist (and thus $\mathbf{H 1}$ is not satisfied) may be given. The following example has the form $X_{n}=f\left(\xi_{n}, \xi_{n+1}\right)$, with $\left\{\xi_{n}\right\}_{n \geq 1}$ a sequence of i.i.d. r.v.'s. Observe that in this case, it is sufficient to construct $X_{1}=f(U, V)$ and $X_{2}=f(V . Z)$. $U, V, Z$ i.i.d., in such a way that there exists an infinity of $u_{n}<v_{n}<\omega$, $u_{n} \nearrow \omega$ for which

$$
P\left\{X_{1} \leq u_{n}, X_{2}>u_{n}\right\}<P\left\{X_{1} \leq v_{n}, X_{2}>v_{n}\right\} .
$$

Let $U$ have strictly positive density on $\mathbb{R}$ and let

$$
X_{1}= \begin{cases}l & \text { if }[V]=2 l+1 . l \in \mathbb{Z} \\ l+1 & \text { if }[V]=2 l\end{cases}
$$

and

$$
X_{2}= \begin{cases}V & \text { if }[Z]=2 l+1, l \in \mathbb{Z} \\ Z+1 & \text { if }[Z]=2 l\end{cases}
$$

Observe that if $u_{n}<u_{n}$ satisfy (3.4), then

$$
\begin{aligned}
P\left\{X_{1} \leq u_{n} . X_{2}>u_{n}\right\}= & P\left\{X_{1}<u_{n} . X_{2}>v_{n}\right\} \\
& +P\left\{X_{1} \leq u_{n}, u_{n}<X_{2} \leq u_{n}\right\}
\end{aligned}
$$

and

$$
\begin{aligned}
P\left\{X_{1} \leq v_{n}, X_{2}>v_{n}\right\}= & P\left\{X_{1} \leq u_{n}, X_{2}>v_{n}\right\} \\
& +P\left\{n_{n} \leq X_{1} \leq v_{n}, X_{2}>v_{n}\right\} .
\end{aligned}
$$

Vol. 34, $n^{\circ}+1998$ 
whence (3.4) is equivalent to

$$
P\left\{X_{1} \leq u_{n} \cdot u_{n}<X_{2} \leq v_{n}\right\}<P\left\{u_{n} \leq X_{1} \leq v_{n}, X_{2}>v_{n}\right\} .
$$

If we take $u_{n}=2 n$ and $v_{n}=2 n+1$, then

$$
P\left\{X_{1} \leq 2 n, 2 n<X_{2} \leq 2 n+1\right\}=0
$$

whereas

$$
\begin{aligned}
& P\left\{2 n \leq X_{1} \leq 2 n+1, X_{2}>2 n+1\right\} \\
& \quad \geq P\{[U]=2 n,[V]=2 n+1 \cdot[Z]=2 n\} \\
& \quad=P\{[U]=2 n\} \times P\{[V]=2 n+1\} \times P\{[Z]=2 n\}>0 .
\end{aligned}
$$

Thus, we have (3.5).

Let

$$
h(u):=1-H(u)
$$

The function $h(u)$ is strictly increasing to 1 for $u_{0} \leq u \leq 1$ (see remark 3.2). We first state our results in terms of the 1 -dependent stationary sequence

$$
Z_{n}=h\left(F\left(X_{n}\right)\right), \quad n \geq 1
$$

whose right end point is 1 .

DEFINITION 3.1. - For any fixed $\rho_{0}, h\left(u_{0}\right) \leq \rho_{0}<1$, define the " $\gamma$-2-block records" of $\left\{Z_{n}\right\},\left\{\left(\tau_{n}, \rho_{n}\right)\right\}_{n \geq 1}$, as follows:

$$
\begin{aligned}
& \tau_{1}=\inf \left\{k \geq 1, Z_{k}>\rho_{0}\right\}, \\
& \rho_{1}=\gamma_{\rho_{0}}\left(\max \left(Z_{\tau_{1}}, Z_{\tau_{1}+1}\right)\right),
\end{aligned}
$$

where for any $\rho_{0} \leq \rho<\approx$,

$$
\gamma_{\mu}(z)=z+P\left\{\xi_{1}>\rho, \rho<\xi_{2} \leq z, \xi_{3}>z\right\}
$$

with $\left(\xi_{1}, \xi_{2}, \xi_{3}\right) \stackrel{\Sigma}{=}\left(Z_{1}, Z_{2}, Z_{3}\right)$.

For $n \geq 1$

$$
\tau_{n+1}=\inf \left\{k>\tau_{n}, Z_{k}>\rho_{n}\right\}
$$

and

$$
\rho_{n+1}=\gamma_{p_{u}}\left(\operatorname{madx}\left(Z_{\tau_{n}} \cdot Z_{\tau_{n-1}}\right)\right)
$$

Remark 3.3. - The sequence $\left\{\left(\tau_{r}, \rho_{n}\right)\right\}$ depends on the choice of the initial threshold $\rho_{0}$. However, we shall prove the following consistency result. 
Proposition 3.1. - Let. $\left\{\left(r_{n}^{\prime}, \mu_{n}^{\prime}\right)\right\}$ be a sequence such that for some $n_{0}, \rho_{n_{0}}^{\prime} \geq h\left(u_{0}\right)$ and for $n \geq n_{0},\left(\tau_{n}^{\prime}, \rho_{n}^{\prime}\right)$ are defined by the recurrence formulas (3.10), in which the $\tau$ and $\rho$ are replaced by $\tau^{\prime}$ and $\rho^{\prime}$.

Then there exists r.v.'s $M$ and $K$ such that for $n \geq M$,

$$
\tau_{n}^{\prime}=\tau_{n-K^{-}} \text {and } \rho_{n}^{\prime}=\rho_{n-K^{\prime}} .
$$

Our main result is the following theorem.

Theorem 3.1. - Let $\left\{X_{n}\right\}$ satisfy hypotheses $\mathrm{H1}$ and H2. Then, there exists a probability space which carries, in addition to $\left\{X_{n}\right\}$, an i.i.d. uniformly on $[0.1]$ distributed sequence $\left\{\dot{Z}_{n}\right\}$. Moreover, there exist two random variables $N$ and $Q$ such that a.s. for $n \geq N$, we have

$$
\tau_{n}=\hat{T}_{n-Q} \text { and } \rho_{n}=\dot{\theta}_{n-Q} \text {. }
$$

with $\left\{\left(\hat{T}_{n}, \hat{\theta}_{n}\right)\right\}$ the record sequence of $\left\{\hat{Z}_{n}\right\}$.

In terms of the initial sequence $\left\{X_{n}\right\}$. we then deduce the following corollary.

Consider the family of functions $\left\{G_{u}: u \in\left[F^{-1}\left(u_{0}\right), \omega\right]\right\}$, where $G_{u}:[u, \omega] \rightarrow[u, \omega]$ is defined as

$$
G_{u}(v)=F^{-1} \circ h^{-1} \circ \gamma_{h \circ F^{\prime}(u)}(h \circ F(v)) . \quad u<v<\omega,
$$

with $\gamma_{\rho}(z)$ defined in (3.9) and $u_{1}$ of remark 3.2. We then have, for

$$
F^{-1}\left(u_{u}\right) \leq u<\omega, G_{u}(u)=u \text { and } G_{u}(v) \geq v, u \leq v<\omega .
$$

For any fixed $\rho_{0}^{\mathrm{X}}, F^{-1}\left(u_{0}\right) \leq \rho_{0}^{\mathrm{X}}<\omega$, define the "G-2-block records" of $\left\{X_{n}\right\},\left\{\left(\tau_{n}^{\mathrm{X}}, \rho_{n}^{\mathrm{X}}\right)\right\}_{n \geq 1}$, as follows:

$$
\begin{gathered}
\tau_{\mathrm{i}}^{\mathrm{X}}=\inf \left\{k \geq 1, X_{k}>\rho_{0}^{\mathrm{X}}\right\}, \\
\rho_{1}^{\mathrm{X}}=G_{\mu_{0} \mathrm{y}}\left(\max \left(X_{\tau_{\mathrm{j}}} \mathrm{x} \cdot X_{\tau_{\mathrm{i}}{ }^{\mathrm{X}}+1}\right)\right) .
\end{gathered}
$$

and for $n \geq 1$

$$
\tau_{n+1}^{X}=\inf \left\{k>\tau_{n}^{X}: X_{k}>\rho_{n}^{X}\right\},
$$

and

$$
\mu_{n+1}^{X}=G_{p_{n}^{\lambda}}\left(\max \left(X_{\tau_{i n}^{X}}, X_{\tau_{i, k}+1}\right)\right) .
$$

Corollary 3.1. - Let $\left\{X_{n}\right\}$ satisfy hypotheses $\mathrm{H1}$ and $\mathrm{H} 2$. Then, there exists a probability space which carries, in addition to $\left\{X_{n}\right\}$ an i.i.d. sequence $\left\{\hat{X}_{n}\right\}_{n \geq 1}$ with marginal distribution

$$
P\left\{\hat{X}_{1} \leq x\right\}=1-P\left\{X_{1} \leq x, X_{2}>x\right\}, x \geq F^{-1}\left(u_{0}\right) .
$$

Vol. 34. $n^{\circ} 4-1998$ 
Moreover, there exist random variables $N$ and $Q$ such that as. for $n \geq N$, we have

$$
\tau_{n}^{\mathrm{X}}=\hat{T}_{n-Q}^{\mathrm{X}} \text { and } \mu_{n}^{\mathrm{X}}=\hat{\theta}_{n-Q}^{\mathrm{X}} .
$$

with $\left\{\left(\dot{T}_{n}^{\mathrm{X}} \cdot \hat{\theta}_{n}^{\mathrm{X}}\right)\right\}$ the record sequence of $\left\{\hat{\mathrm{X}}_{n}\right\}$.

DEFINITION 3.2. - For any fixed $\mathcal{T}_{0}, h\left(u_{0}\right) \leq \mathcal{T}_{0}<1$, define the 2 block record sequence of $\left\{Z_{n}\right\}$ with respect to the initial threshold $\mathcal{T}_{0}$, $\left\{\left(\mathcal{T}_{n} \cdot \mathcal{R}_{n}\right)\right\}_{n>1}$, ass

$$
T_{1}=\inf \left\{K_{i} \geq 1: Z_{k}>T_{1}\right\} . \quad R_{1}=\max \left(Z_{T_{1}} \cdot Z_{T_{1}+1}\right)
$$

and for $n \geq 1$

$$
\mathcal{T}_{n+1}=\inf \left\{k>\mathcal{T}_{n}: Z_{k}>\mathcal{R}_{n}\right\}
$$

and

$$
\mathcal{R}_{n+1}=\max \left(Z_{T_{n+1}}, Z_{T_{n+1}+1}\right) .
$$

$\mathcal{T}_{n}$ are the 2-block record times and $\mathcal{R}_{n}$ the 2-block record lalues.

Remark 3.4. - Let $\left\{\left(\mathcal{T}_{n}^{\prime}, \mathcal{R}_{n}^{\prime}\right)\right\}$ be a sequence such that for $n \geq n_{0}, \mathcal{T}_{n}^{\prime}$ and $\mathcal{R}_{n}^{\prime}$ are defined by the recurrence formulas (3.16). in which $\mathcal{T}$ and $\mathcal{R}$ are replaced by $\mathcal{T}^{\prime}$ and $\mathcal{R}^{\prime}$. Then. it can be easily seen that there exist $m$ and $h$ such that for $n \geq m$,

$$
\mathcal{T}_{n}^{\prime}=\mathcal{T}_{n-k} \text { and } \mathcal{R}_{n}^{\prime}=\mathcal{R}_{n-k} .
$$

The next result is the following theorem 3.2.

THEOREM 3.2. - If $\left\{X_{n}\right\}$ satisfies hypotheses $\mathbf{H} 1$ and $\mathbf{H 2}$, then, there exist r.v.'s $M$ and $L$ such that a.s. for $n \geq M$, we have

$$
\tau_{n}=\mathcal{T}_{n-1 .} \text { and } \mathcal{R}_{n-L} \leq \rho_{n} \leq \mathcal{R}_{n-L+1} \leq \rho_{n+1} .
$$

The analogous result holds for the sequence of 2-block records of $\left\{X_{n}\right\}$. $\left\{\left(\mathcal{T}_{n}^{\mathrm{Y}}, \mathcal{R}_{n}^{\mathrm{Y}}\right)\right\}$ and $\left\{\left(\tau_{n}^{\mathrm{X}}, \rho_{n}^{\mathrm{Y}}\right)\right\}$, i.e.

$$
\tau_{n}^{\mathrm{X}}=\mathcal{T}_{n-L}^{\mathrm{X}} \text { and } \mathcal{R}_{n-L}^{\mathrm{X}} \leq \rho_{n}^{\mathrm{X}} \leq \mathcal{R}_{n-L+1}^{\mathrm{X}} .
$$

Remark 3.5. - Combining (3.12) and (3.18), we also may say that there exist r.v.'s $M^{\prime}$ and $L^{\prime}$ such that a.s. for $n>M^{\prime}$, we have

$$
\hat{T}_{n}=\mathcal{T}_{n-L^{\prime}} \text { and } \mathcal{R}_{n-L^{\prime}} \leq \hat{\theta}_{n} \leq \mathcal{R}_{n-L^{\prime}+1} \leq \hat{\theta}_{n+1} .
$$


This in particular means that the 2-block record limes of $\left\{X_{n}\right\}$ (resp. $\left.\left\{Z_{n}\right\}\right)$ a.s. assymptotically coincide via an index translation to the record times of an i.i.d. sequence $\left\{\hat{X}_{n}\right\}$ (resp. $\left\{\hat{Z}_{n}\right\}$ ) and that the 2-block record values of $\left\{X_{n}\right\}$ (resp. $\left\{Z_{n}\right\}$ ) and the record values of $\left\{\hat{X}_{n}\right\}$ (resp. $\left\{\hat{Z}_{n}\right\}$ ) are imbricate.

In example 5 of section 2, we have $Z_{n}=1-X_{n}^{2}+X_{n}^{3}=X_{n}+$ $O\left(\left(1-X_{n}\right)^{2}\right)$ and the 2-block records of $\left\{X_{n}\right\}$ a.s. coincide, via an index translation, to the records of the i.i.d. sequence $\left\{U_{n+1}\right\}$. This behaviour does not contradict the statements of Theorems 3.1 and 3.2.

We now prove proposition 3.1 and theorems 3.1 and 3.2 by means of the following intermediary results.

LEMMa 3.1. - There exists a probability space which carries, in addition to $\left\{X_{n}\right\}$, a sequence $\left\{\left(S_{n}, R_{n}\right)\right\}_{n>1}$ having the same Markov structure as the records of an i.i.d. sequence $\left\{\hat{Z}_{n}\right\}$ with $U[0.1]$ distributed margins, i.e. for any $n \geq 0$

$1 \leq t_{1}<t_{2}<\ldots<t_{u}, s \geq 1$ and $s_{0}=u_{0}<u_{1}<\ldots<u_{n+1}<1$,

$P\left\{S_{n+1}-S_{n}=s_{;} R_{n+1}<u_{n+1} \mid S_{1}=t_{1}, R_{1}=u_{1} ; \ldots ; S_{n}=t_{n}, R_{n}=u_{n}\right\}$ $=P\left\{S_{n+1}-S_{n}=s: R_{n+1}<u_{n+1} \mid R_{n}=u_{n}\right\}$ $=u_{n}^{*-1}\left(u_{n+1}-u_{n}\right)$.

Furthermore, there exists a r.l. $N$ such that a.s. for $n \geq 1, S_{n}$ and $R_{n}$ are defined by the recurrence formulas of the $\gamma$-2-block records, i.e.:

$$
\begin{aligned}
& S_{n+1}=\inf \left\{k>S_{n}: Z_{k}>R_{n}\right\} \\
& \text { and } R_{n+1}=\gamma_{R_{n}}\left(\max \left(R_{T_{n+1}}, R_{T_{n+1}+1}\right)\right) .
\end{aligned}
$$

Remark 3.6. - Note that the sequence $\left\{\left(S_{n}, R_{n}\right)\right\}$ depends on the particular choice of the initial threshold $u_{0}$.

Proof. - By (Haiman (1987a), théorème 1', p. 432), there exist $0<u_{1}<1$, a constant $C_{1}$ and a function $\mu(u), 0<\mu(u)<1$, such that

$$
\begin{aligned}
& \max _{n \geq 1}\left|\frac{P\left\{\max \left(Z_{1} \ldots Z_{n}\right) \leq u\right\}}{\mu^{\prime \prime}(u)}-1+P\left\{Z_{1}>n, Z_{2}>u\right\}\right| \\
& \quad \leq C_{1}\left(P\left(Z_{1}>u\right)\right)^{2} .
\end{aligned}
$$

Furthermore, there exist constants $C_{2}$ and $C_{3}$ such that, for $u_{1} \leq u \leq 1$, we have

$$
\begin{aligned}
& \left|\mu(u)-\left(1-P\left\{Z_{1} \leq u, Z_{2}>u\right\}\right)\right| \\
& \quad=|\mu(u)-u| \leq C_{2}\left(P\left(Z_{1}>u\right)\right)^{2} \leq C_{3}(1-u)^{2} .
\end{aligned}
$$

Vol. $34, n^{\circ} 4-1998$. 
Remark 3.7. - In the sequel we take for $u_{0}$ the maximum of $u_{0}$ in remark 3.2 and $u_{1}$.

For $k \geq 2$ and $u_{0} \leq u<v<1$, let

$$
\begin{aligned}
\mathcal{A}_{k} & =\mathcal{A}_{k}(u, d v) \\
& =\left\{Z_{1} \leq u, \ldots, Z_{k-1} \leq u . Z_{k}>u, \gamma_{u}\left(\max \left(Z_{k}, Z_{k+1}\right)\right) \in(n ; v+d v]\right\} .
\end{aligned}
$$

It may be checked that

$P\left\{\mathcal{A}_{2}(u, d v)\right\}=P\left\{Z_{1} \leq u, Z_{2}>u, \gamma_{u}\left(\max \left(Z_{2}, Z_{3}\right)\right) \in(v ; v+d v]\right\}=d v$.

Next,

$P\left\{\mathcal{A}_{3}\right\}$

$=P\left\{\mathcal{A}_{2}\right\}-P\left\{Z_{1}>u, Z_{2} \leq u, Z_{3}>u, \gamma_{u}\left(\max \left(Z_{3}, Z_{4}\right)\right) \in(v ; v+d v]\right\}$,

and, for $k \geq 4$,

$$
\begin{array}{r}
P\left\{\mathcal{A}_{k}\right\}=P\left\{Z_{1} \leq u, \ldots, Z_{k-3} \leq u\right\} \cdot P\left\{\mathcal{A}_{2}\right\} \\
-P\left\{Z_{1} \leq u, \ldots, Z_{k-3} \leq u, Z_{k-2}>u, Z_{k-1} \leq u,\right. \\
\left.Z_{k}>u, \gamma_{u}\left(\max \left(Z_{k}, Z_{k+1}\right)\right) \in(v ; v+d v]\right\},
\end{array}
$$

where. by stationarity and 1-dependence, the second term is majorizated by

$$
\begin{aligned}
& P\left\{Z_{1} \leq u, \ldots, Z_{k-4} \leq u\right\} \cdot P\left\{Z_{1}>u\right\} \\
& \quad \cdot P\left\{Z_{1}>u, \gamma_{u}\left(\max \left(Z_{1}, Z_{2}\right)\right) \in(v ; v+d v]\right\}
\end{aligned}
$$

(by convention, $P\left\{Z_{0} \leq u\right\}=1$ ). Now, by hypothesis $\mathbf{H 2}$,

$$
P\left\{Z_{1}>u \cdot \gamma_{u}\left(\max \left(Z_{1}, Z_{2}\right)\right) \in(v ; v+d v]\right\}=O_{1}\left(P\left\{\mathcal{A}_{2}\right\}\right), u \rightarrow 1 .
$$

Thus, combining (3.21), (3.24)-(3.28), and observing that $u=1-(1-u)$, we obtain, for $k \geq 2$,

$$
P\left\{\mathcal{A}_{k}(u, d v)\right\}=u^{k-1} d v\left(1+O_{2}\left((1-u)^{2}\right)\right)^{k-1}\left(1+O_{3}(1-u)\right),
$$

where $\left|O_{i}(x)\right| \leq C_{i}|x|$ with $C_{i}, i=1.2,3$ universal constants. We shall now construct $\left\{\left(S_{n}, R_{n}\right)\right\}$ by using recursively the following lemma.

LEMmA 3.2. (Haiman (1987a), p. 448). - Let $Y$ a random variable taking values in a measurable space $(\mathcal{Y}, \mathcal{F})$. Let $\varphi \in \mathcal{F}$ and let $\hat{P}$ be a probability 
measure on $(\mathcal{Y}, \mathcal{F})$ such that $0<\hat{P}(\varphi)<1$. Assume that on $\varphi$, the probability distribution of $Y$, denoted $P_{Y}$, has a Radon-Nicodym derivative $\frac{d P_{Y}}{d \vec{P}}$ with respect to $\hat{P}$ such that

$$
\max _{y \in \varphi}\left|\frac{d P_{Y}}{d \hat{P}}(y)-1\right|(1-\hat{P}(\varphi))^{-1} \leq q<1 .
$$

Let $Q$, be a Bernoulli $r . v$. independent of $Y$ such that $P(Q=0)=q$. Then there exist two random variables $Y^{\prime}$ and $\bar{Y}$, taking values respectively in $\mathcal{Y}$ and $\varphi^{\prime}$, independent of $Y$ and $Q$, and such that, if we put

$$
\hat{Y}= \begin{cases}Y & \text { si } Q=1 \text { et } Y \in \varphi \\ Y & \text { si } Q=1 \text { et } Y \in \varphi^{\prime} \\ Y^{\prime} & \text { si } Q=0\end{cases}
$$

then, the probability distribution of $\hat{Y}$ is $\hat{P} .\left(\varphi^{\circ}\right.$ denotes the complementary event of $\varphi$.)

Let $S_{0}=u_{0}$ and define $\left(S_{1}, R_{1}\right)$ independently of $\left\{X_{n}\right\}_{n \geq 1}$. Moreover, suppose that for $1 \leq p \leq n,\left(S_{p} . R_{p}\right)$ have allready been constructed and that the events of the form $\left\{\left(S_{1}=s_{1}, R_{1} \in \mathcal{A}_{1}\right), \ldots,\left(S_{n}=s_{n}, R_{n} \in \mathcal{A}_{n}\right)\right\}$ (where $1 \leq s_{1}<\ldots<s_{n}$ and $\mathcal{A}_{i}, 1 \leq i \leq n$, are Borel sets of $\mathbb{R}$ ) are $\sigma\left\{X_{1}, \ldots, X_{s_{n}+1}\right\} \times \sigma^{\prime}$ measurable, where $\sigma^{\prime}$ is a $\sigma$-field independent of $\sigma\left\{X_{n}, n \geq 1\right\}$

Let $Y=Y_{s, r}, r \geq u_{0}$. be the random variable taking values in $\mathcal{Y}=\{1,2 \ldots\} \times(r, 1)$, defined conditionaly given $S_{n}=s$ and $R_{n}=r$ as follows:

$$
Y_{s, r}=\left(1, r^{\prime}\right), r<r^{\prime}<1 \Leftrightarrow\left\{Z_{s+3}>r, \gamma_{r}\left(\max \left(Z_{s+3}, Z_{s+4}\right)\right)=r^{\prime}\right\}
$$

and, for $t \geq 2$,

$$
\begin{gathered}
Y_{*, r}=\left(t, r^{\prime}\right) . r<r^{\prime}<1 \text { if and only if } \\
\left\{Z_{s+3} \leq r, \ldots, Z_{s+2+t-1} \leq r, Z_{s+2+t}>r, \gamma_{r}\left(\max \left(Z_{s+2+t}, Z_{s+3+t}\right)\right)=r^{\prime}\right\}
\end{gathered}
$$

Let $\hat{P}$, be the probability measure on $\mathcal{Y}$, defined conditionaly given $S_{n}=s$ and $R_{n}=r$ by

$$
\hat{P}(t,[v, n+d v])=r^{t-1} d v, \quad t \geq 1, r<v<1 .
$$

Let

$$
\varphi=\varphi_{r}=\left\{\left(t, r^{\prime}\right) \in \mathcal{Y}: 2 \leq t \leq[\psi(r)]\right\}
$$

Vol. $34, n^{2} 4-1998$ 
where

$$
\psi(r)=\frac{\tau}{1} r \ln _{2}\left(\frac{1}{1-r}\right), u_{0}<r<1 .
$$

with $\tau>1$ a fixed constant and $\ln _{2}(x)=\ln (\ln x)$. We then have

$$
\hat{P}\left(\varphi_{i}^{\prime}\right)=1-\dot{P}(\varphi)=1-r+r^{[\varphi(r)]}>r^{[\varphi(r)]} \sim\left(\ln \left(\frac{1}{1-r}\right)\right)^{-\tau} \cdot r \rightarrow 1 .
$$

Next, denoting by $Y_{1}, Y_{2}$ the coordinates of $Y_{s, r}=Y$, observe that

$$
P\left\{Y_{1}=l, Y_{2} \in[v, v+d v]\right\}=P\left\{\mathcal{A}_{k}(u, d v)\right\} .
$$

Thus, combining (3.29), (3.32)-(3.38) and using the fact that $(1+x)^{k}=$ $1+O(k x) . r \rightarrow 0$, we obtain

$$
\max _{y \in \varphi^{\prime}}\left|\frac{d P_{Y}}{d \hat{P}}(y)-1\right|(1-\hat{P}(\varphi))^{-1} \leq c(1-r)\left(\ln \left(\frac{1}{1-r}\right)\right)^{\tau^{\prime}}=q(r) .
$$

for some constants $C>0$ and $\tau^{\prime}>\tau$. Since $q(r) \rightarrow 0$ as $r \rightarrow 1$, for $r \geq u_{2}$ large enough (from now one we take for $u_{0}$ the maximum of $u_{0}$ in remark 3.7 and $u_{2}$ ), we may apply lemma 3.2.

Let $Q_{n+1}=Q$ with $Q$ of the lemma (i.e. $P\left(Q_{n+1}=0\right)=q\left(R_{n}\right)$ ).

Let $L_{n+1}$ be a Bernoulli r.v. such that

$$
P\left(L_{n+1}=1\right)=r^{2} .
$$

Furthermore, let $Q_{n+1}$ and $L_{n+1}$ be mutually independent and depend on $\left\{X_{n}\right\}$ and $\left(S_{1}, R_{1}\right), \ldots\left(S_{n}, R_{n}\right)$ only through $R_{n}$.

Let $\dot{Y}=\left(\tilde{Y}_{1}, \dot{Y}_{2}\right)$, be a random vector taking values in $\{1,2\} \times\left(R_{n}, 1\right)$ such that

$$
P\left\{\tilde{Y}_{1}=t: \tilde{Y}_{2} \leq v\right\}=r^{t-1}(v-r) \frac{1}{P\left(L_{n+1}=0\right)}, t=1.2 . r<v<1 .
$$

(We take $\dot{Y}$ independent of the previously defined random elements.) We now define $\left(S_{n+1}, R_{n+1}\right)$ as follows:

If $L_{n+1}=0$. then

$$
S_{n+1}=S_{n}+\dot{Y}_{1} \text { and } R_{n+1}=\grave{Y}_{2} .
$$

If $L_{n+1}=1$, then

$$
S_{n+1}=S_{n}+2+\dot{Y}_{1} \text { and } R_{n+1}=\dot{Y}_{2} \text {. }
$$


where $\left(\hat{Y}_{1}, \hat{Y}_{2}\right)=\hat{Y}$ is given by lemma 3.2 .

It is not difficult to check that $\left\{\left(S_{1}, R_{1}\right) \ldots,\left(S_{n+1}, R_{n+1}\right)\right\}$ satisfy (3.19) and the measurability conditions requested previously at order $n$ also at order $n+1$.

Now, in order to show that there exists a r.v. $N$ such that a.s. for $n \geq N$, relation (3.20) is satisfied, it is sufficient to prove, denoting by $E_{n}$ the event

$$
E_{n}=\left\{L_{n+1}=1 .\left(\max \left(Z_{S_{n+1}+}, Z_{S_{n+3}}\right)<R_{n}\right), Q_{n+1}=1 . Y^{\prime} \in \varphi R_{n}\right\} .
$$

that we have

$$
P^{\prime}\left\{E_{n}^{c} \text { i.o. }\right\}=0
$$

where $E_{n}^{c}$ is the complementary event of $E_{n}$. In order to obtain (3.45), we will prove that

$$
\begin{aligned}
& P\left\{L_{n+1}=0 \text { i.o. }\right\}=P\left\{\max \left(Z_{S_{n}+2}, Z_{S_{n}+3}\right)>R_{n} \text { i.o. }\right\}= \\
& P\left\{Q_{n+1}=0 \text { i.o. }\right\}=P\left\{Y \in \varphi_{R_{n}}^{c} \text { i.o. }\right\}=0 .
\end{aligned}
$$

Remark 3.8. - Observe that if $\left\{\left(S_{n}, R_{n}\right)\right\}$ satisfies (3.19), then it is possible to construct an i.i.d. uniformly on [0.1] distributed sequence $\left\{\hat{Z}_{n}\right\}$ such that $\left(S_{n}, R_{n}\right)$ is the record sequence of $\left\{\hat{Z}_{n}\right\}$ with respect to the initial threshold $u_{0}$.

Hence, by (Haiman (1987), Lemma 3, p. 454), for any $0<1<<1$, we have

$$
P\left\{1-R_{n}>e^{-\alpha \prime \prime} \text { i.o. }\right\}=0
$$

and for any $\beta>1$.

$$
P\left\{1-R_{n}<e^{-i 3 n} \quad \text { i.o. }\right\}=0 .
$$

Next, if $A_{n}$ is one of the events in the brackets in (3.46), $P\left\{A_{n}\right.$ i.o. $\}=0$ is equivalent to $P\left\{A_{n} \cap\left(1-R_{n} \leq t^{-t n}\right)\right.$ i.o. $\}=0$.

Let

$$
B_{n}=\left(L_{n+1}=0\right) \cap\left(1-R_{n} \leq e^{-1 n}\right) .
$$

We then have from (3.39)

$$
P\left(B_{n}\right)=\int_{1 \ldots, \ldots n}^{1}\left(1-r^{2}\right) d P_{R_{n}}(r) \leq e e^{-\alpha n}
$$

Vol. $34, n^{2}+1998$ 
with $c$ positive constant, which is the general term of a convergent series. Thus

$$
P\left(L_{n+1}=0 \text { i.o. }\right)=0
$$

follows by the first Borel-Cantelli lemma.

In a similar way, we obtain

$$
P\left\{\left(Q_{n+1}=0\right) \cap\left(1-R_{n} \leq e^{-\alpha n}\right)\right\} \leq c^{\prime} e^{-\alpha n}(\alpha n)^{\tau^{\prime}}, 1<\tau<\tau^{\prime},
$$

and

$$
P\left\{\left(Y \in \varphi_{R_{n}}^{c}\right) \cap\left(1-R_{n} \leq^{-\alpha n}\right)\right\} \leq c^{\prime \prime} n^{-\tau} .
$$

from which

$$
P\left(Q_{n+1}=0 \text { i.o. }\right)=P\left\{Y \in \varphi_{R_{n}}^{c} \text { i.o. }\right\}=0
$$

follows. Let now

$$
C_{n}=\left(Q_{n+1}=1\right) \cap\left(L_{n+1}=1\right) \cap\left(Y \in \varphi_{R_{n}}^{i}\right) \cap\left(1-R_{n} \leq e^{-c x n}\right)
$$

and

$$
D_{n}=C_{n} \cap\left\{\max \left(Z_{S_{n}+2}, Z_{S_{n}+3}\right)>R_{n}\right\} .
$$

We then similarly obtain

$$
P\left(D_{n}\right)<c e^{-o n} \ln (n)
$$

and thus the last equality in (3.45). This achieves the proof of lemma 3.1.

Proof of Proposition 3.1 and Theorems 3.1 and 3.2. - In order to prove proposition 3.1 and theorems 3.1 and 3.2 , we also need the following lemmas:

Lemma 3.3. - Consider, with $\left\{\left(S_{n}, R_{n}\right)\right\}$ of lemma 3.l, the events

$$
\begin{aligned}
\mathcal{A}_{n} & =\left\{\inf \left[m>S_{n+1}+2 ; Z_{m}>\max \left(Z_{S_{n+1}}, Z_{S_{n+1}+1}\right)\right]<S_{n+2}\right\} \\
& =\bigcup_{m \geq 2}\left\{\left[\max \left(Z_{S_{n+1}+2}, \ldots, Z_{S_{n+1}+m}\right) \leq \max \left(Z_{S_{n+1}}, Z_{S_{n+1}+1}\right)\right]\right. \\
& \left.\cap\left[Z_{S_{n+1}+m+1} \in\left(\max \left(Z_{S_{n+1}}, Z_{S_{n+1}+1}\right), \gamma_{R_{n}}\left(\max \left(Z_{S_{n+1}}, Z_{S_{n+1}+1}\right)\right)\right]\right]\right\} .
\end{aligned}
$$

Then

$$
P\left\{\mathcal{A}_{n} \text { i.o. }\right\}=0 \text {. }
$$

Remark 3.9. - Lemma 3.3 implies that there exists a r.v. $M$ such that a.s. for $n \geq M$.

$$
S_{n+1}=\inf \left\{k>S_{n} ; Z_{k}>\max \left(Z_{S_{n}}, Z_{S_{n}+1}\right)\right\} .
$$

i.e. for $n \geq M$ the $S_{n}$ are given by the recurrence formulas of the 2-block record times of $\left\{Z_{n}\right\}$ in (3.16). 
Proof of Lemma 3.3. - Let, taking into account (3.46) and (3.47),

$$
\mathcal{B}_{n}=\mathcal{A}_{n} \cap\left(1-R_{n} \leq^{-\alpha n}\right) \cap\left(1-R_{n+1}>e^{-\beta n}\right) .
$$

We then have

$$
\begin{aligned}
P\left(\mathcal{B}_{n}\right)= & \sum_{t=2 n+1}^{\infty} \iint_{د} \\
& \times P\left\{\mathcal{A}_{n} \mid R_{n}=r, S_{n+1}=t, R_{n+1}^{\prime}=s\right\} d P_{\left(R_{n}, S_{n+1}, R_{n+1}^{\prime}\right)}(r, t, s),
\end{aligned}
$$

where

$$
\Delta=\left\{(r, s) ; u_{0} \leq r<s<1,1-r \leq^{-\alpha n)}: 1-s>e^{-\beta n}\right\}
$$

and

$$
R_{n+1}^{\prime}=\max \left(Z_{S_{n+1}} \cdot Z_{S_{n+1}+1}\right) .
$$

Thus

$$
\begin{aligned}
& P\left\{\mathcal{A}_{n} R_{n}=r, S_{n+1}=t, R_{n+1}^{\prime}=s\right\} \\
& \quad \leq P\left\{\bigcup_{m \geq 3}\left(\max \left(Z_{t+3}, \ldots, Z_{t+m}\right)<s\right) \cap\left(Z_{t+m+1} \in\left(s, \gamma_{r}(s)\right]\right)\right\} \\
& \quad \leq K_{1} P\left\{Z_{1} \in\left(s, \gamma_{r}(s)\right]\right\} \sum_{k=0}^{\infty} \mu^{k}(s)
\end{aligned}
$$

with, by (3.22),

$$
\mu(s)=s+O\left((1-s)^{2}\right) .
$$

Thus, we easily deduce that there exists a positive constant $K_{2}$ such that

$$
P\left\{\mathcal{A}_{n} \mid R_{n}=r, S_{n+1}=l, R_{n+1}^{\prime}=s\right\} \leq K_{2} \frac{\gamma_{r}(s)-s}{1-s} .
$$

Next, by (3.9)

$$
\begin{aligned}
\gamma_{r}(s)-s & =P\left\{\xi_{1}>r, r<\xi_{2} \leq s, \xi_{3}>s\right\} \\
& \leq P\left\{\xi_{1}>r\right\} \cdot P\left\{\xi_{3}>s\right\}=O\left((1-r)^{2}\right) .
\end{aligned}
$$

Thus, there exists a constant $K_{3}$ such that on $\Delta$

$$
P\left\{\mathcal{A}_{n} \mid R_{n}=r, S_{n+1}=t, R_{n+1}^{\prime}=s\right\} \leq K_{3} \frac{e^{-2 \alpha n}}{e^{-\beta n}}=K_{3} e^{-(2 \alpha-\beta) n} .
$$

Vol. 34, $n^{\circ} 4-1998$. 
Choose $\alpha$ and $\beta$ such that $2 \varepsilon-\beta>0$. Then

$$
P\left\{\mathcal{B}_{n}\right\} \leq K_{3} r^{-(2 n-3) n}
$$

which is the general term of a convergent series and we get

$$
P\left\{\mathcal{B}_{\text {ri.o. }}\right\}=0 \text {. }
$$

whence $(3.50)$.

Remark 3.10. - Let $\left\{\left(\tau_{n}^{\prime}, \rho_{n}^{\prime}\right)\right\}_{n \geq 1}$ be any sequence such that for $n \geq n_{0}$, $\left(\tau_{n}^{\prime}, \rho_{n}^{\prime}\right)$ are defined by the recurrence formulas of the $\gamma$-2-block records (3.10).

We then observe that either there exist $N_{1}$ and $Q_{1}$ such that for $n \geq N_{1}$

$$
\tau_{n-Q_{1}}^{\prime}=S_{n} \text { and } \rho_{n-Q_{1}}^{\prime}=R_{n} \text {. }
$$

or there exist $N_{2}$ and $Q_{2}$ such that for $n \geq N_{2}$

$$
S_{n}<\tau_{n-Q_{2}}^{\prime}<S_{n+1} \text { and } R_{n}<\rho_{n-Q_{2}}^{\prime}<R_{n+1} .
$$

Thus, by (3.46) and (3.47), we also have:

for any $0<a<1$

$$
P\left\{1-\tau_{n}^{\prime}>r^{-n n} \text { i.o. }\right\}=0
$$

and for any $\beta>1$

$$
P\left\{1-\tau_{n}^{\prime}<1^{-3 n} i .0 .\right\}=0 .
$$

Let $\mathcal{A}^{\prime}$ " be the event defined by formula (3.49), in which $S_{n+1}, S_{n+2}$ are replaced by $\tau_{n+1}^{\prime}, \tau_{n+2}^{\prime}$ and $R_{n}$ by $\rho_{n}^{\prime}$. We then may use the proof of lemma 3.3. without any other changes, to deduce that

$$
P\left\{\mathcal{A}_{n}^{\prime} \text { i.r. }\right\}=0 \text {. }
$$

But this implies that there exists a r.v. $M^{\prime}$, such that a.s. for $n \geq M^{\prime}$

$$
\tau_{n+1}^{\prime}=\inf \left\{h>\tau_{\prime \prime}^{\prime}: Z_{k}>\max \left(Z_{\tau_{\prime \prime}^{\prime}} \cdot Z_{\tau_{n}^{\prime}+1}\right)\right\} .
$$

Then, by remark 3.4, there exist r.v.'s $M^{\prime}$ and $K^{\prime \prime}$ such that a.s. for $n \geq M^{\prime}$

$$
\tau_{n}^{\prime}=S_{n-K^{\prime}} \text { and } \rho_{n}^{\prime}=R_{n-K^{\prime \prime}}
$$

This proves proposition 3.1. The proof of theorem 3.1 also readily follows by taking $\left\{\hat{Z}_{n}\right\}$ the i.i.d. sequence of remark 3.8, $\hat{T}_{n}:=S_{n}$ and $\hat{\theta}_{n}:=R_{n}$. Remark 3.9 and theorem 3.1 then imply theorem 3.2. 


\section{AC KNOWLEDGMENT}

We wish to thank Professor J. Bretagnolle for giving us a first counterexample which inspired the one presented in remark 3.2 and Professor M.A. Lifshits for his remark leading to theorem 1.2.

\section{REFERENCES}

[1] G. HaImAN, Etude des éxurômes d'une suile stationnaire m-dépendante avec une alplication relative aux accroissements du processus de Wiener., Annales de l'institut Henri Poincaré. Vol. 23, 1987a, $\mathrm{N}^{\circ}$ 3. p. $425-458$.

[2] G. Haiman, Almost stre asymptotic behaviour of the record and record time sequences of a stationary gaussian pricess. Mathematical Statistics and Probability Theory. vol. A. 1987b. p. $105-120$.

131 G. HaIMAN. A strong invariance principle for the extremes of multivariate stationary m-dependent sequences. Journal of Statistical Planning and Inference, Vol. 32. 1992. p. $47-163$.

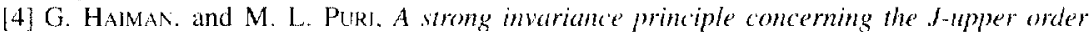
statisfics for stationary Geussian sequences. Annals of Probability. Vol. 21, N $1,1993$. p. $86-135$.

[5| G. Haiman, M. KJKı and M. L. Purı, Extreme of Marker sequences, Journal of Statistical Planning and Inference. Vol. 45. 1995, p. 185-201.

[6| F. MATUS, On nor-blork-fictor sequences and one dependence. Preprint of the Institute of Information Theory and Automation, Academy of Sciences of the Czech Republic. 1994.

[7] N. MaYelr, Thèse de Doctoral, 1996.

[8] V. Nevzorov. Records. Theory Probah.Appl., Vol. 32, N 2, 1987, p. 201-22X.

(Manuscript received November 29. 1996;

Revised wersion rectived Manh 20, 1998.) 